\title{
Validation of a new approach for distinguishing anesthetized from awake state in patients using directed transfer function applied to raw EEG
}

\author{
Bjørn E. Juel ${ }^{1,2} \mathbb{E} \cdot$ Luis Romundstad $^{3} \cdot$ Johan F. Storm ${ }^{1} \cdot$ Pål G. Larsson $^{4}$
}

Received: 29 June 2020 / Accepted: 1 October 2020 / Published online: 16 October 2020

(c) The Author(s) 2020

\begin{abstract}
We test whether a measure based on the directed transfer function (DTF) calculated from short segments of electroencephalography (EEG) time-series can be used to monitor the state of the patients also during sevoflurane anesthesia as it can for patients undergoing propofol anesthesia. We collected and analyzed 25-channel EEG from 7 patients ( 3 females, ages 41-56 years) undergoing surgical anesthesia with sevoflurane, and quantified the sensor space directed connectivity for every 1-s epoch using DTF. The resulting connectivity parameters were compared to corresponding parameters from our previous study ( $n=8$, patients anesthetized with propofol and remifentanil, but otherwise using a similar protocol). Statistical comparisons between and within studies were done using permutation statistics, a data driven algorithm based on the DTF-parameters was employed to classify the epochs as coming from awake or anesthetized state. According to results of the permutation tests, DTF-parameter topographies were significantly different between the awake and anesthesia state at the group level. However, the topographies were not significantly different when comparing results computed from sevoflurane and propofol data, neither in the awake nor in anesthetized state. Optimizing the algorithm for simultaneously having high sensitivity and specificity in classification yielded an accuracy of $95.1 \%$ ( $\mathrm{SE}=0.96 \%$ ), with sensitivity of $98.4 \%$ $(\mathrm{SE}=0.80 \%)$ and specificity of $94.8 \%$ ( $\mathrm{SE}=0.10 \%)$. These findings indicate that the DTF changes in a similar manner when humans undergo general anesthesia caused by two distinct anesthetic agents with different molecular mechanisms of action.
\end{abstract}

Keywords Anesthesia monitoring $\cdot$ Electroencephalography $\cdot$ EEG $\cdot$ Directed transfer function $\cdot$ Measure of consciousness

\section{Introduction}

Objective quantification of how EEG signals change in relation to subjects' states of consciousness has a long history [1-3]. Recently, measures quantifying properties such as complexity [4], functional and effective connectivity [5],

Bjørn E. Juel

b.e.juel@medisin.uio.no

1 Brain Signaling Group, Institute of Basic Medical Science, University of Oslo, Oslo, Norway

2 Department of Psychiatry, Center for Sleep and Consciousness, University of Wisconsin - Madison, Madison, USA

3 Department of Anesthesiology, Rikshospitalet, Oslo University Hospital, Oslo, Norway

4 Department of Neurosurgery, Rikshospitalet, Oslo University Hospital, Oslo, Norway and information content [6] in signals recorded with electroencephalogram (EEG) have been used successfully for objectively distinguishing between conscious and apparently unconscious states in humans. Generally, an apparent loss of consciousness is related to changes in such EEG signal properties or a combination of them [7], but capturing the relevant changes in a way that makes the measures useful for bedside monitoring of the level of consciousness in patients is not straightforward.

Recently, we published results indicating that the directed transfer function (DTF) - a measure that can be used to quantify sensor space directed connectivity from EEG recordings of spontaneous brain activity—changed abruptly as patients undergoing surgical propofol anesthesia apparently lost and regained consciousness [8]. The observed changes could be used to classify the state of individual patients as awake or anesthetized with $98 \%$ accuracy with a temporal resolution of $1 \mathrm{~s}$. Importantly, these results were obtained using EEG data recorded in a normal clinical 
setting (general anesthesia for surgery), and without requiring any data cleaning, indicating that the DTF may be useful for developing objective, real-time monitors of patients undergoing anesthesia.

The previous study was done on a population of patients undergoing a single anesthetic protocol during surgerypropofol anesthesia with the opioid analgesic remifentanil. Thus, it is conceivable that the differences between the awake and anesthetized state observed in that study merely reflected changes related to the specific anesthetic agent rather than the changes related to general anesthesia, or loss of consciousness, in general. If so, the DTF-based method may not be fit to distinguish between states of consciousness more generally. However, qualitatively similar findings have been reported when using DTF to assess brain connectivity in groups of patients suffering from disorders of consciousness [9], and healthy individuals falling asleep [10-12]. Taken together, this suggests that the DTF calculated from EEG may consistently change between conscious and unconscious states, regardless of how the change in state of consciousness came about.

Here, we report from a follow-up study, designed to test whether the DTF-based approach presented in our previous study can also be used to distinguish between awake and anesthetized states in patients undergoing general anesthesia caused by sevoflurane. Thus, we test whether the changes observed in the DTF derived connectivity parameters in the propofol study, were also apparent for patients undergoing sevoflurane anesthesia, and whether the changes could once again be used to successfully classify the state of the patients in accordance with the clinician's judgement of their state of wakefulness.

\section{Methods}

\subsection{Study design}

This was a single-center observational study designed to investigate how the volatile anesthetic sevoflurane affects particular DTF derived connectivity parameters calculated from EEG recordings of spontaneous brain activity. The EEG data were collected from patients undergoing surgical sevoflurane anesthesia with fentanyl. The data were collected between September 2016 and February 2017 in experiments performed at the Oslo University Hospital, Rikshospitalet. Patients were recruited by the surgeon in charge of all included surgeries, the same surgeon as in our previous study [8]. The study was approved by the Regional Committee for Research Ethics (case number 2012/2015), and all patients included in the study signed a written consent form after oral and written information.

\subsection{Inclusion and exclusion criteria}

As in our previous study, the patients included were scheduled for anterior cervical discectomy and fusion, and the surgery was performed under total intravenous general anesthesia. The patients were (1) American Society of Anesthesia I-III patients (ASA Physical Status Classification System. American Society of Anesthesiologists; https://www.asahq.org/resources/clinical-information/ asa-physical-status-classification-system) (2) between 18 and 60 years old, and (3) seen as otherwise healthy based on a complete health examination. Patients were excluded if they had known hypersensitivity to sevoflurane or fentanyl, any history of, or family members with, malignant hyperthermia, soy oil or egg allergy, liver or renal disease affecting drug pharmacodynamics, heart or lung disease causing physical limitations (unable to climb two stairs without rest), body mass index $>30 \mathrm{~kg} / \mathrm{m}^{2}$, any impaired general health condition from abuse of drugs and alcohol, organ damage, or neurological or psychiatric disease. In total, 8 patients were recruited and underwent the anesthetic and surgical procedures required for the study.

\subsection{Anesthetic management}

The patients fasted for at least $6 \mathrm{~h}$ before anesthesia. Their premedication consisted of oral paracetamol (Paracet ${ }^{\circledR}$, Weifa, Oslo, Norway) 1.5 g, midazolam (Dormicum ${ }^{\circledR}$, Basel, Switzerland) $3.75-7.5 \mathrm{mg}$ for mild sedation, and oxycodone sustained release tablet (opioid analgesic; OxyContin ${ }^{\circledR}$, Dublin, Ireland) $10 \mathrm{mg}$. Premedication was given $45 \mathrm{~min}$ before anesthesia. Before induction of anesthesia, an infusion with Ringer Acetat was started to compensate for any hypotension caused by anesthesiainduced vasodilatation and cardiodepression during induction. During anesthesia, the patients were monitored with pulse-oximetry (SpO2), and measurements of end tidal carbon dioxide (ETCO2), end tidal sevoflurane concentration (ETsevo), electrocardiography (ECG), and oscillometric noninvasive blood pressure (BP) every $5 \mathrm{~min}$. Anesthesia was induced with sevoflurane gas delivered via a vaporizer coupled to a semi-open breathing system and led to the patient through a tight face mask. The drugs used for anesthesia were sevoflurane, a non-pungent, nonirritable, ultra-short acting halogenated volatile general anesthetic (Sevofluran ${ }^{\circledR}$, Baxter Medical AB Kista Sweden) and fentanyl $50 \mu \mathrm{g} / \mathrm{ml}$, a potent, short-acting synthetic opioid analgesic (Fentanyl®, Hameln Pharmaceuticals Hameln Germany). After pre-oxygenation with $100 \%$ oxygen $10 \mathrm{l} / \mathrm{min}$ for $3 \mathrm{~min}$ with spontaneous breathing in a tight face mask, fentanyl was given intravenously, and the 
sevoflurane vaporizer was set at maximum concentration of $8 \%$. As the patient's wakefulness and respiratory drive declined, the anesthesiologist started carefully to assist the ventilation with $8 \%$ sevoflurane in $100 \%$ oxygen $(10 \mathrm{ml} /$ min). When loss of eyelash reflex was observed, and the EEG had changed character from dominant alpha and low beta band activity to strong delta and theta/alpha activity in the frontal electrodes, the patient was intubated with an endotracheal tube. No neuromuscular blockers were used in the intubation process, except in one patient (\#7) who required it for a smooth intubation. As soon as correct placement of the tube was verified, mechanical ventilation with $5 \%$ sevoflurane in medical air with $40 \%$ oxygen in nitrogen was started and the fresh gas flow was reduced from 10 to $21 / \mathrm{min}$. We then varied the sevoflurane concentration between 5 and $3 \%$ aiming for an end tidal sevoflurane concentration between 3 and 2\% and 1-1.5 minimum alveolar concentration (MAC). Nitrous oxide was not used. All the patients received local anesthetic infiltration with $5 \mathrm{ml} \mathrm{5 \%}$ bupivacaine in the area of the skin incision.

\subsection{Assessment of consciousness}

The patients' state of wakefulness was assessed clinically by the anesthesiologist throughout the surgical procedure using standard anesthetic tools and practices. During the anesthesia induction phase, the Modified Observer's Assessment of Alertness/Sedation Scale (MOAAS) [13] was used to measure the patient's state of wakefulness until loss of verbal contact and loss of response was reached. The MOAAS assessment was employed by the anesthesiologist maintaining verbal communication with the patient, and the patients were considered anesthetized and unconscious when they did no longer respond to their name being called (MOAAS level 2). At this point, the MOAAS assessment was discontinued, until the patient was about to wake up again. Throughout maintenance of anesthesia, the state of the patient was monitored clinically (by observing the heart rate, blood pressure, sweating, tear production, eye and eyelid reflexes, pupil size and symmetry, and any limb movements) with standard clinical equipment to ensure the conditions were suitable for all stages of surgery. Furthermore, the raw EEG, especially the recordings from the frontal electrodes, were observed providing information regarding the depth of anesthesia [2]. Time points for initiation and discontinuation of sevoflurane administration, loss of consciousness (LOC, i.e. corresponding to loss of verbal contact and behavioral response), and return of verbal communication (ROC) were recorded immediately by the electrophysiologist monitoring the EEG.

\subsection{EEG methods}

EEG was recorded for the duration of the clinical procedure, including segments before, during, and after anesthesia. In total, 25 passive electrodes were used, 19 of which were placed in accordance with the 10-20 system (no mastoid electrodes), with six additional electrodes positioned to capture lower lateral activity (F9, F10, T9, T10, P9, P10). CP1 was used as the recording reference. No re-referencing was performed during the analysis.

The processing steps applied to the data closely resembled the analysis pipeline described in our previous paper [8]. It should be noted that the precise choices made for preprocessing may impact the results of the analysis. Therefore, in line with the aim of the study, we opted to stay as close as possible to the pre-processing used in our original study and leave exhaustive exploration of the effects of preprocessing to future work. Each patient's EEG data was read into Matlab using BioSig as implemented in EEGLAB [14], and cut into non-overlapping 1-s epochs. The epochs from before LOC and after ROC were labeled as coming from the awake state, while the epochs between the markers for LOC and ROC were labeled as coming from the anesthetized state. Before further analysis, the data for each patient were automatically scanned for artefactual epochs and channels using a simple in-house algorithm based on the statistics of the patient's own EEG signal. An epoch was marked as artefactual if it had large (deviating by more than 3 standard deviations from the median calculated from the patient's own typical epochs signal) peak-to-peak amplitude, high variance, or transient currents (sudden changes in voltage) within the epoch. Similarly, a channel was marked as artefactual if its peak-to-peak amplitude, variance, or transient currents were different (again, deviating by more than 3 standard deviations from the median) when compared to other channels across epochs in the same patient's EEG signal. The artefacts were not removed for the analyses but were labeled and used to indicate likely artefacts in figures (e.g. white patches in Figs. 4, 6).

For all epochs, the relevant DTF variables were calculated (see $[8,15])$. The DTF is a Granger causality type, multivariate directed functional connectivity measure, which can be computed for multichannel time series data such as EEG. It is computed by taking the Z-transform of the coefficients of an autoregressive model of the data [17]. The resulting DTF parameters, quantifying the connectivity from one channel to another, are then normalized by the sum of incoming connectivity to the receiving channel, so that the values of the connectivity falls in the range $[0,1]$. Thus, a high value indicates a strong directed connectivity, while low values indicate that there is a weak (or no) directed connectivity between the channels. For a more complete and mathematical exposition of the DTF, please see [16, 17]. 
DTF was calculated for each $1 \mathrm{~s}$ epoch independently in the theta frequency range $(4-8 \mathrm{~Hz})$, using the DTF function from the eConnectome toolbox [18]. The median of the resulting matrices of DTF values was calculated across frequencies, yielding a typical strength of information flow between every pair of EEG electrodes in the theta band. The logarithm was taken to more clearly distinguish between small DTF values resulting in our main measure, referred to as LDTF. We also calculated the LDTF source strength (or information outflow) by taking the median across all outgoing connections from a given EEG channel. This was called mLDFT (or median information outflow) and represents the typical information each EEG channel apparently contains about the future activity in the other channels.

The LDTF values of each accepted epoch was then classified as coming from a segment recorded during the 'awake' or 'anesthetized' state using the classification algorithm presented in our previous work [8]. The algorithm was based on a leave-one-out cross-validation scheme. This means that, for each patient, the LDTF values from each epoch were compared to the 'awake' and 'anesthetized' distributions of LDTF values from all other patients. The two distributions were generated by independently pooling the LDTF values from epochs marked as coming from the 'awake' and 'anesthetized' state. Thus, the LDTF values from a given patient and epoch were compared with the distributions of LDTF values from the other patients. The comparison yielded a value indicating the likelihood, $\mathrm{L}_{\text {state }}$, that the LDTF values from a given epoch were drawn from the awake $\left(\mathrm{L}_{\text {awake }}\right)$ or anesthetized ( $\left.\mathrm{L}_{\text {anesthetized }}\right)$ state. The classification of the epoch, as either 'anesthetized' or 'awake', depends on the relationship between the likelihood values related to the two conditions. To quantify this relationship, we defined the 'classification confidence' (or 'confidence of classifying an epoch as coming from the awake distribution'), $\mathrm{C}$.

$C=\frac{L_{\text {awake }}}{L_{\text {awake }}+L_{\text {anesthetized }}}$,

Here, $\mathrm{L}_{\text {awake }}$ is the likelihood that the LDTF values of the epoch were drawn from the (empirical) distribution of LDTF values from all other patients in the awake state. Similarly, $\mathrm{L}_{\text {anesthetized }}$ is the likelihood that the LDTF values of the epoch were drawn from the (empirical) distribution of LDTF values from all other patients in the anesthetized state.

We classified patients as awake or anesthetized in a given 1-s epoch depending on whether their C-value for that epoch was bigger or smaller than a preset threshold. To find the optimal value for this threshold, we varied the threshold between zero and one, and calculated the accuracy, sensitivity, and specificity of classification for each threshold value. The optimal value for $\mathrm{C}$ was defined as the threshold that produced the maximum sum of sensitivity and specificity across all patients.

\subsection{Propofol experiment}

We directly compared the results of our analysis of the patients undergoing sevoflurane anesthesia to the same analysis applied to data from patients undergoing propofol anesthesia recorded for our previous study [8]. Here, we give a brief description of the protocol used in that study, but for a complete description, please consult the original report.

Ten patients scheduled to undergo anterior cervical discectomy and fusion were recruited by their handling doctor to be included in a single-center observational study of patients undergoing general propofol anesthesia with remifentanil. Inclusion and exclusion criteria were similar to the present study. Of the eight patients analyzed in our previous study (one excluded due to data quality, and one due to falling asleep in the "awake" period), the first seven were included in this study to keep the samples of equal size between the study populations. Premedication protocol, monitoring of the patient state throughout the surgical procedure, and the EEG equipment used was as described above. The patients received propofol anesthesia with remifentanil which was delivered using target controlled infusion, and the anesthesia had a median duration of $163 \mathrm{~min}$.

\subsection{Statistics}

The topographical maps of LDTF values were compared between states within each anesthetic protocol (awake vs anesthetized), as well as between types of anesthesia (sevoflurane vs propofol (from our previous study)). To do this, we calculated the $\mathrm{mLDTF}_{\text {awake }}$ and $\mathrm{mLDTF}_{\text {anesthesia }}$ for every patient (including patients from our previous study), and used permutation statistics method described by Karniski et al. [19] to compare the topographical maps between states and anesthetics. In brief, we quantified the difference between the mLDTF topographies (spatial maps of median information outflow from EEG channels) observed in the awake and anesthetized state, using the T-sum-squared statistic suggested by Karniski et al. Then, we made all possible permutations of groupings (switching the labels of mLDTF $_{\text {awake }}$ and mLDTF $_{\text {anesthesia }}$ for the patients in all possible ways) and quantified the difference between each of the groups using the same test statistic. If $<5 \%$ of the permuted groups showed larger differences than the original grouping (e.g. awake vs anesthesia), the difference was considered significant. This was done for the sevoflurane patients as well as the propofol patients. We also did a permutation test with the data from propofol and sevoflurane pooled together, to compare the mLDTF topographies between states (awake and anesthetized) irrespective of anesthetic agent. 
To investigate whether the changes observed under sevoflurane were comparable to changes observed under propofol, we ran two more permutation tests. Since the patients in the propofol study differ from the patients in the current study the permutation tests were repeated 100 times, each time with a different ordering of the participants. This was done because the permutation test comparison compares the topographies in a pairwise manner to compute the test statistics. Therefore, repeated tests with shuffled order of patients were required to avoid any bias of the arbitrary ordering of patients. In this way, we compared the mLDTF topographies in the propofol data (from previous study) with those in the sevoflurane data (from this study) within the awake and anesthetized conditions separately. This was done in order to test the hypothesis that the mLDTF topographies in comparable behavioral states were not different between the two experiments.

\section{Results}

The final data material comprised 7 patients ( 3 female) with a median age of 48 years (range $41-56$ years). One patient (patient \#6) was excluded from the analysis because large portions of the data were corrupted. During the induction phase, the patients were intravenously given $350 \mu \mathrm{g}$ (range $250-450 \mu \mathrm{g}$ ) of the analgesic drug fentanyl, and the percentage of sevoflurane in the end-tidal volume was measured and adjusted (measured range 2.6-5.8\%, mean $4.2 \%$ ). After the patients became unresponsive no further doses of fentanyl were given. The median sevoflurane concentration during the maintenance phase of anesthesia was measured to $2.0 \%$ (range 1.7-2.4\%) of end-tidal volume. Throughout the surgical procedure, physiological variables followed the expected development during anesthetic induction, maintenance, and emergence (Fig. 1).

From each of the patients, continuous EEG was recorded throughout the clinical procedure (median length: $161 \mathrm{~min}$, range 115-247 $\mathrm{min}$ ). Every recording contained segments from before (median length: $9 \mathrm{~min}$, range 7-16 min), during (median length: $148 \mathrm{~min}$, range 105-232 $\mathrm{min}$ ), and after anesthesia (median length: $3 \mathrm{~min}$, range $2-5 \mathrm{~min}$ ). In the automatic artefact scanning process, $13 \%$ (range 2-39\%) of all epochs from the wakeful periods were marked as artefactual, while $6 \%$ (range $4-17 \%$ ) of epochs were marked from the anesthesia period. In addition, a variable number of channels (median: 2, range 0-4) channels were marked as artefactual.

The DTF analysis showed a qualitative difference between the 'awake' and 'anesthetized' states. The mLDTF topography was heterogeneous in the 'awake' state, but more homogenous in the 'anesthetized' state (Fig. 2b). A relatively strong apparent source of information outflow could be seen located over the posterior midline channels, whereas this region was far less distinct in the plot for the 'anesthetized' state. Qualitatively speaking, this looks similar to the changes observed in our previous work with patients undergoing propofol anesthesia (Fig. 2c), and lends support to our previous finding that the awake state is associated with a more heterogeneous pattern of outgoing information flow than the anesthetized state.

The mLDTF topographies from each individual, in both awake and anesthetized states, can be seen in Fig. 3. These topographies show the median LDTF values for each patient, across all epochs in a particular state ('awake' or 'anesthesia'). Permutation tests indicate that the qualitative differences observed between conditions were statistically significant ( $\mathrm{p}=0.016 ; 2^{7}=128$ permutations) for the patients undergoing sevoflurane anesthesia. A significant change was also observed when comparing the awake with the anesthetized condition in the data from the patients undergoing propofol anesthesia $\left(\mathrm{p}=0.016 ; 2^{7}=128\right.$ permutations). This was also the case when pooling together the data from the two experiments, indicating that the differences observed here might be similar to those observed in our previous study $\left(\mathrm{p}=4.0^{*} 10^{-4} ; 2^{14}=16,384\right.$ permutations $)$. In all tests, the natural grouping of patient labels (anesthesia vs awake) was the grouping with the largest overall difference between the groups of all possible permutations, indicating the strongest possible evidence for differences between groups given the number of samples. Furthermore, when comparing within conditions, between experiments (propofol anesthetized vs sevoflurane anesthetized and propofol awake vs sevoflurane awake), the test yielded non-significant results ( $p>0.05$ for all 100 runs; $2^{7}=128$ permutations). Thus, there was little or no evidence for differences between mLDTF values in the patient groups from the two studies, in either the anesthetized or the awake state, and we cannot reject the hypothesis that the mLDTF topographies in comparable behavioral states were not different between the two experiments.

The mLDTF patterns remained relatively stable over time within states, but of the patterns transitioned abruptly when the patients' state of wakefulness changed (Fig. 4). This was the case both when patients transitioned from wakefulness to anesthesia (Fig. 4, left column) as well as when the patient regained consciousness after anesthesia (Fig. 4, right column). However, there was no distinct change in the mLDTF pattern around the time when the anesthesia delivery was stopped (Fig. 4, middle column). Importantly, the abrupt change in mLDTF pattern was clearly reflected in the algorithm's 'classification confidence' (red lines in Fig. 4), which appears to transition between two distinct states right around the transitions between wakefulness and anesthesia.

From the LDTF values, the algorithm's confidence (C) in classifying a patient as awake in a given one-second epoch was calculated to form a basis for classification. Varying 
a
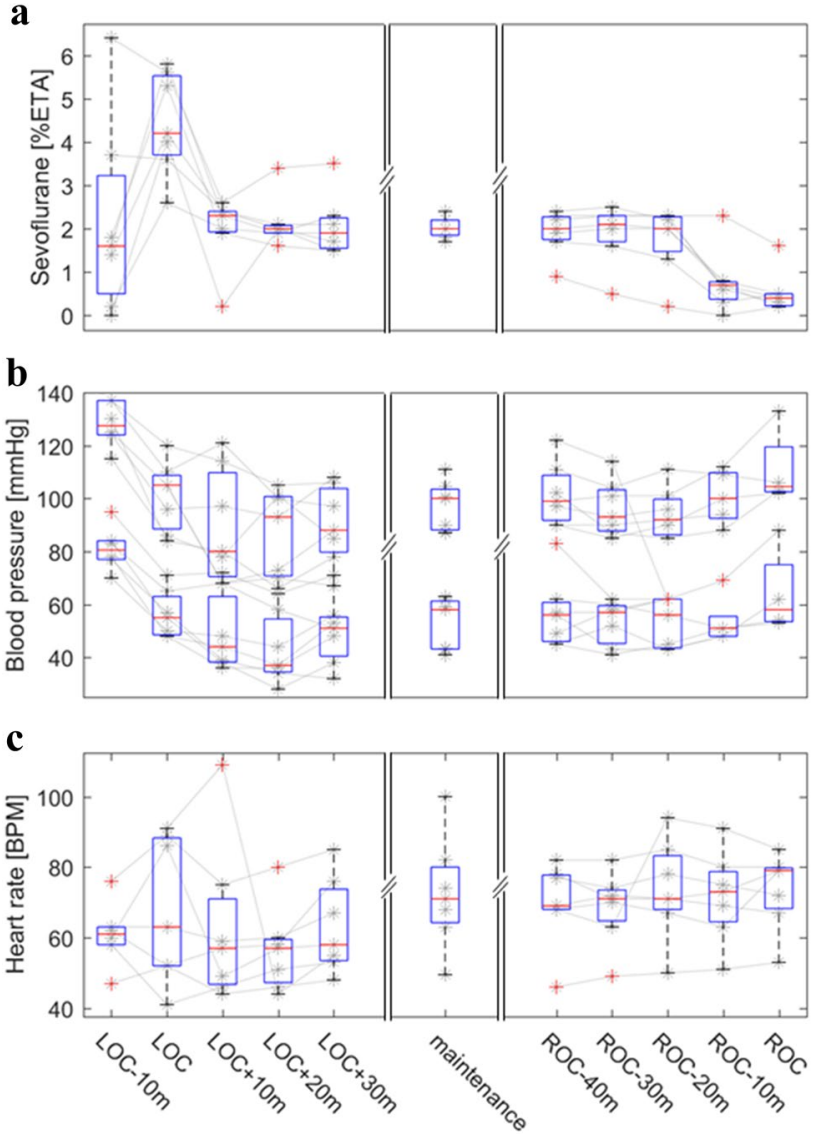

Fig. 1 End-tidal sevoflurane concentration, blood pressure, and heart rate measurements during induction, maintenance, and re-emergence phases of surgical procedure. The panels show the relevant measures for each patient (gray lines and stars) with a box-and-whisker plot overlaid, summarizing the population statistics (red line: median, box: interquartile range, whiskers: minimum to maximum value, red crosses: outliers). Panel a shows the changes in percentage of sevoflurane in the end-tidal volume. Panel $\mathbf{b}$ shows systolic and diastolic blood pressure values. Panel c shows the heart rates. Each panel is subdivided into three parts, indicating the dynamics of each measurement during induction to (left) and reemergence from (right) anesthesia, and the mean values recorded throughout the maintenance phase (middle). The left and right parts of the panels (induction and reemergence) show values from individual measurements of each patient, measured every $10 \mathrm{~min}$

the threshold value required for classifying a patient as awake resulted in a maximum overall accuracy of $96.8 \%$ $(\mathrm{SE}=0.63 \%)$ for threshold values of $0.1<\mathrm{C}<0.17$ (Fig. 5). While sensitivity monotonically increases, and specificity monotonically decreases, for larger cut-off values, the classification accuracy remained high for a broad range of threshold values, only dropping below $96 \%$ for $\mathrm{C}<0.01$ and $\mathrm{C}>0.9$. However, when we optimized for maximized sum of sensitivity and specificity, the range of valid threshold values shrank significantly, and moved towards lower values of $\mathrm{C}$. Specifically, the optimal threshold value was
$\mathrm{C}=0.001$, yielding an accuracy of $95.1 \%(\mathrm{SE}=0.001)$, with sensitivity of $98.4 \%(\mathrm{SE}=0.80 \%)$ and specificity of $94.8 \%$ ( $\mathrm{SE}=0.10 \%)$. The results of the classification using the optimal threshold value is shown in Fig. 6 to give an impression of the temporal stability of the mLDTF patterns and the accuracy of the classification for every patient.

\section{Discussion}

This study shows that our DTF-based measure yields similar results when applied to EEG recordings obtained from patients undergoing general anesthesia with sevoflurane and with propofol. Like we found with propofol anesthesia in our previous paper [8], the source strength topographies went from having relatively stronger apparent sources of information outflow in the posterior region of the head in the 'awake' state, to becoming far more homogeneously distributed in the 'anesthetized' state during sevoflurane anesthesia (Fig. 3). The differences between conditions (awake vs. anesthesia) were significant for both anesthetics (propofol and sevoflurane), and there was no significant difference between the changes observed with the two anesthetics. Taken together, this indicates a common change being captured by the LDTF when comparing the awake and anesthetized conditions, using different anesthetics, under slightly different conditions, and using slightly different analysis. Finally, the changes in DTF-based parameters could be successfully used to classify the patients' states as awake or anesthetized in accordance with the clinician's judgement with a 1-s temporal resolution.

Even though these results are similar to what was found in our previous study, there were some differences in the analysis that should be mentioned. In addition to adding an automatic artefact-scan (only used for visualization/reporting), we changed two aspects in the analysis: in the present study we (1) focused on the theta rather than alpha frequency band, and (2) optimized the classification using the parameter $\mathrm{C}$ - the 'confidence of classifying an epoch as coming from the awake distribution'.

We changed the frequency band used for DTF analysis from the alpha band, which we used in our previous study [8] to the theta band, to reduce potential confounds caused by changes in the alpha band power related to closing of the eyes (patients had their eyes open in the awake state but closed in the anesthetized state). The patterns of DTF derived connectivity observed in the two bands were relatively similar in our previous analyses leading us to believe that it would be possible to successfully use the theta band for the purpose of distinguishing between the states. Indeed, this change seems to yield a small benefit in reducing one potential confound, without causing any clear disadvantages or detrimental effects to the classification. Similarly, the 


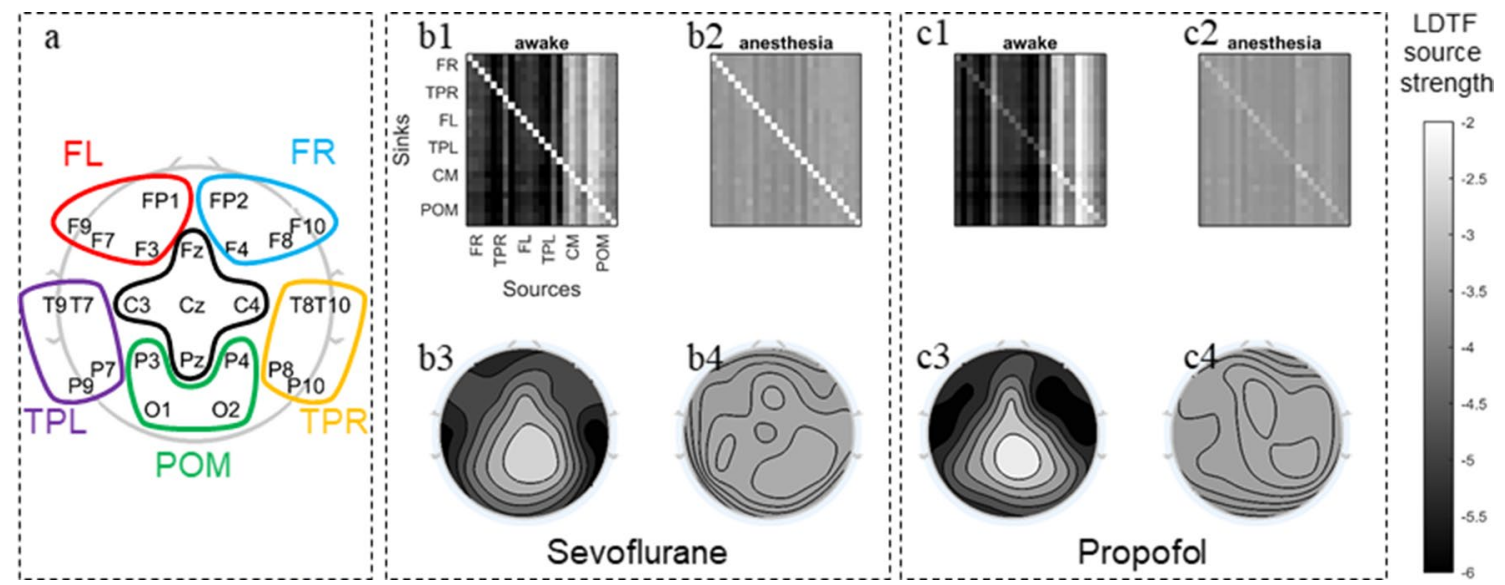

Fig. 2 Summary figure showing population median DTF connectivity patterns. Panel a shows a topographical representation of the channel montage used during the EEG recordings. For clarity, sets of electrodes grouped together and marked by colors in order to simplify the displays in the other two panels (Frontal Right (FR) electrodes are marked in Blue: FP2, F4, F8, and F10; Temporoparietal Right (TPR) electrodes are marked in Orange: T8, T10, P8, and P10; Frontal Left (FL) electrodes are marked in Red: FP1, F3, F7, and F9; Temporoparietal Left (TPL) electrodes are marked in Yellow: T7, T9, P7, and P9; Central Medial (CM) electrodes are marked in Black: Fz, $\mathrm{Cz}, \mathrm{C} 4, \mathrm{C} 3$, and Pz; and Posterior Occipital Medial (POM) marked in Green: $\mathrm{Pz}, \mathrm{P} 4, \mathrm{P} 3, \mathrm{O} 2$, and $\mathrm{O} 1)$. In panels $\mathbf{b}$ and $\mathbf{c}$ the population median DTF in the theta range is visually summarized for the sevoflurane (new data) and propofol (data from [8]), respectively. Panels b1 and c1 show the full directed connectivity matrices in the awake state, while b2 and c2 show the same for the 'anesthetized' state. Each element in the matrix quantifies the median information flow from a source channel (x-axis) to a sink channel (y-axis). b2 and c2 show that the DTF-parameter values are very similar across channels, as indicated by the homogeneous color throughout the plots. In the corresponding topographical plots $(\mathbf{b 3}, \mathbf{b 4} ; \mathbf{c 3}, \mathbf{c 4})$, the distribution of information flow sources across the scalp are shown. $\mathbf{b 3}$ and $\mathbf{c 3}$ visualize how DTF-based information sources are distributed across the scalp in the awake state. b4 and c4 do the same for information sources in the anesthetized state. The color scale on the right indicates the relation between the shade used and the values of LDTF for all panels: dark shades indicate strong, while light shades indicate weak, information flow choice of optimizing the threshold parameter, C, seemed to improve the analysis compared to use the more naïve approach used for classification from our previous paper. Interestingly, this optimization had quite a strong effect on the quality of classification in the current paper. Even though the accuracy of classification would have been similar using the naïve approach, the sensitivity was improved when using the optimized threshold (see Fig. 5). In fact, the sensitivity would have been worse than in our previous paper if we had not used a different threshold (only $\sim 70 \%$ of the epochs from awake patients would have been correctly classified, in contrast to the $95 \%$ in our previous paper). However, using the optimized threshold, $\mathrm{C}=0.001$, instead yielded classification results that were slightly better than in our previous study [8].

Another difference from the previous study was that the patients were asked about whether they experienced anything during the anesthesia and surgical procedure, to investigate whether any of them would have recollection of waking up, dreaming, or otherwise being conscious. However, to avoid inducing traumatic memories, the depth of the questioning was kept to a minimum. None of the patients reported memories of waking up during the surgery, but two patients (\#1 and \#8) reported having had simple dreams during the anesthesia. However, their timelines of typical outgoing information flow (mLDTF) did not show any sign of awake-like patterns during the anesthesia. There might be several reasons for this, but we do not have sufficient data to make strong conclusions here. For example, our measure may be insensitive to the dream state, the dreams may have occurred during emergence or have been confabulated, or the patients may have had brief periods of waking up that were later interpreted as dreaming or forgotten. Determining the real causes of this sort of observations requires a different type of protocol, better suited for experiments outside of clinical surgery setting. In other studies, the presence of dreams has been seen to be quite common during general anesthesia, being reported to occur up to $60 \%$ of the time with multiple anesthetics commonly used in clinics, including sevoflurane and propofol $[20,21]$. Whether or not an anesthesia monitor should distinguish between states with and without dreams depends on the purpose of the monitor. Whereas such a distinction may not be relevant for monitors intended to help clinicians determine whether a patient is in a state of general anesthesia suitable for surgery, it may be central for monitors intended to distinguish brain states with and without consciousness defined as experience, including dreams [22, 23].

Of course, the most profound difference between this study and the previous was the fact that the patients 


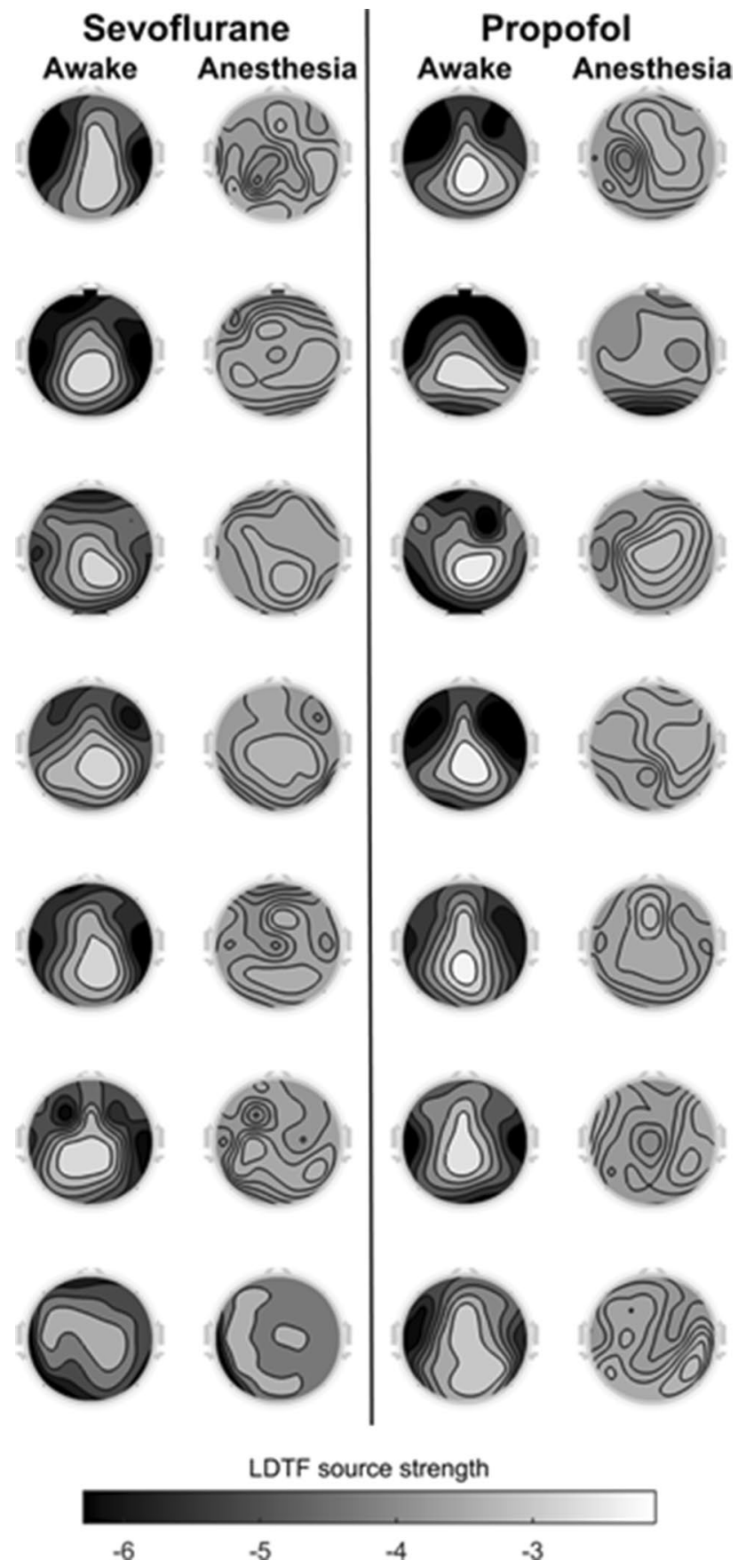

Fig. 3 Topographical maps of DTF-based median outgoing connectivity strengths in the awake and anesthetized state. The topographical map of mLDTF from the sevoflurane (left, theta range mLDTF) and propofol (right, alpha range mLDTF; data from [8]) studies are shown. Each panel shows the pooled mLDTF map for a given patient (row) in a given state (column). Thus, the topographies in the 'awake' column are generated by taking the median LDTF values across all epochs marked by the anesthesiologist as 'awake' for a given participant. Similarly, the topographies in the 'anesthesia' column are based on the median LDTF values across all epoch marked as 'anesthetized'. The color scale on the bottom indicates the relation between the shades used and the values of LDTF for all panels: dark shades indicate strong, while lighter shades indicate weak, DTF-based information flow underwent a different type of anesthesia (sevoflurane rather than propofol). This fact was used to investigate whether the DTF-method can successfully classify the state of wakefulness in patients using an anesthetic with assumed distinct mechanism of action [24, 25], but a comparable endpoint for the patient: unresponsiveness and apparent unconsciousness due to the general anesthetic [26]. The two anesthetics, propofol and sevoflurane, share some mechanisms of action: they both potentiate $\mathrm{GABA}_{\mathrm{A}}$ and glycine receptors, and inhibit voltage gated potassium channels and acetylcholine receptors [24, 25]. However, they also differ in certain respects. Most notably, sevoflurane potentiates two-pore potassium channels and inhibits serotonin receptors, while propofol potentiates kainate receptors. Furthermore, sevoflurane has been reported to have a stronger inhibiting effect on AMPA and NMDA receptors. In short, the two anesthetics have complex and different interactions with ion channels and receptors affecting several neuronal populations, thus profoundly altering the neuronal activity patterns in the brain [27].

These alterations in neuronal activity are reflected in changes in EEG patterns. For example, as the molecular targets and effects of sevoflurane and propofol both partly overlap and partly differ, there are both similarities and differences also between their effects on large-scale measures of brain function [28]. For example, both sevoflurane and propofol have been reported to induce coherent frontal alpha oscillations and slow oscillations in EEG of humans [29], but sevoflurane has also be shown to be unassociated with the typical anteriorization of alpha rhythms [30]. Sevoflurane anesthesia has also been seen to increase the coherence in the theta frequency range $(4-7 \mathrm{~Hz})$, relative to comparable levels of propofol anesthesia [29]. In addition, propofol and sevoflurane have been shown to differentially suppress the relative glucose metabolic rate in several brain regions [31]. Furthermore, it is well known that anesthetics affect somatosensory evoked EEG potentials in humans and animals [32], but sevoflurane affects these potentials more strongly, in a dose-dependent fashion, than comparable doses of propofol [33]. These findings indicate that certain large-scale properties of the EEG do indeed change differentially in response to the two anesthetics. The fact that both the molecular, cellular, and large-scale brain effects of the two anesthetics differ in so many ways, increases the value of testing our method with both compounds, and enhances the significance of the remarkable similarity in their effects on DTF. Thus, since it is not obvious that EEG-derived measures such as the DTF would yield so similar results in patients undergoing anesthesia with as mechanistically different agents as propofol and sevoflurane, our results suggest that they have some substantial large-scale effects in common that are captured by the DTF derived measure. 


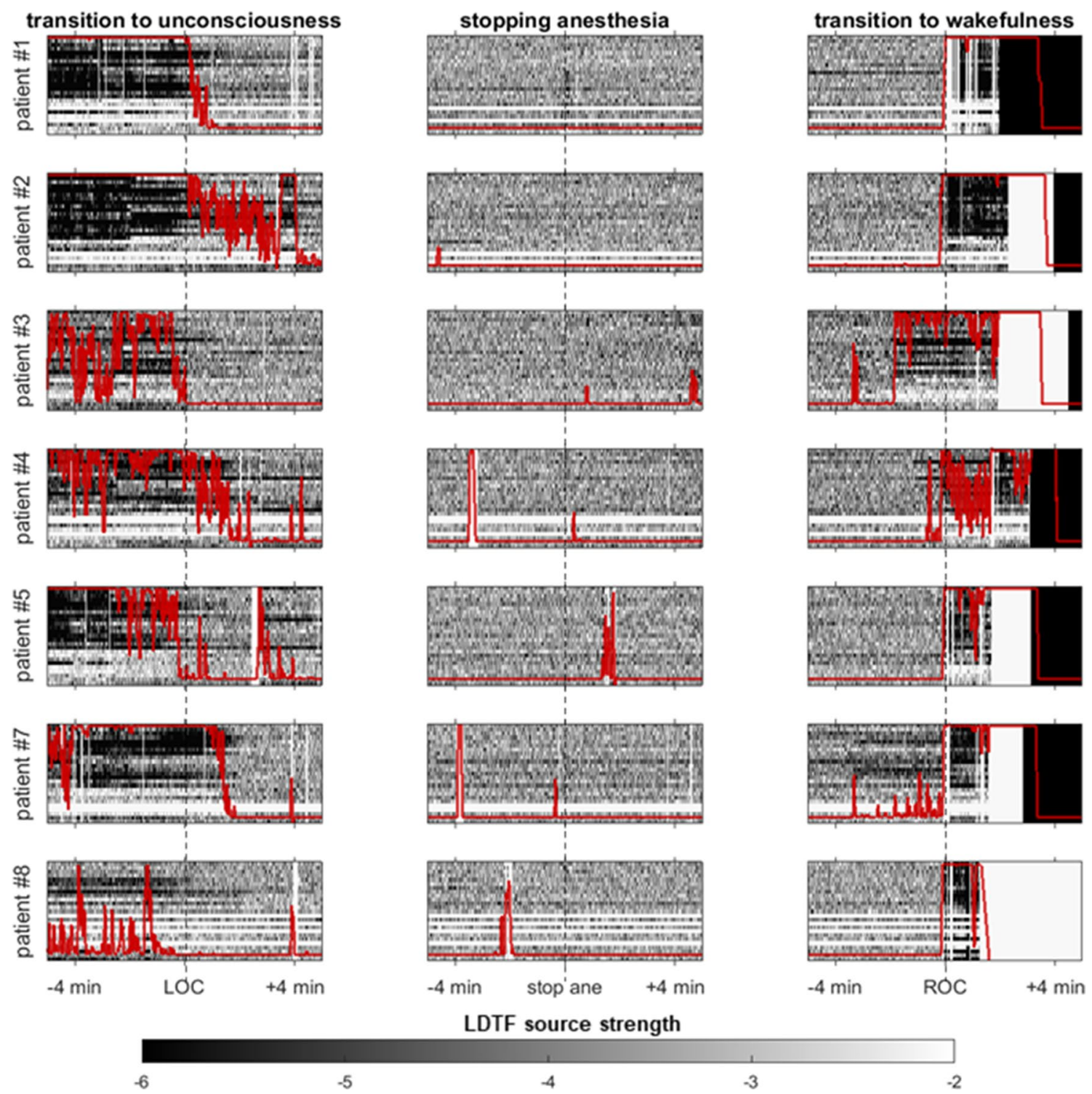

Fig. 4 Visualizing the LDTF source strengths, and the classification confidence, near main events in the anesthetic management. Each row in this figure contains three plots useful for describing the quality of the classification algorithm. The figures in each row shows how the information source strengths change for each patient around three critical points in the anesthetic management-loss of verbal communication (LOC, left), stopping the anesthetic administration (stop ane, centre), and the time of return of verbal communication (ROC, right).
Each plot is time locked to the time-point noted by the clinical staff, and shows the development of the mLDTF source strengths from $5 \mathrm{~min}$ before, to $5 \mathrm{~min}$ after the event. Red lines show the behavior of the classification confidence measure (y-axis ranges from 0 to 1 (bottom to top)). White vertical lines indicate an artefactual epoch, and white horizontal lines indicate an artefactual channel. The black, gray, and white shading in all plots relates to the strength of the information source as indicated by the color bar at the bottom

signals [34]. And, in addition to our own previous study [8], at least four studies have previously investigated how DTFbased connectivity measures are affected by changing states of consciousness [9-12]. Each one of them reported changes in apparent brain connectivity related to distinct physiological states such as different stages of sleep and disorders of consciousness (DOC). Thus, changes in DTF may capture properties related to loss of consciousness in general, not 


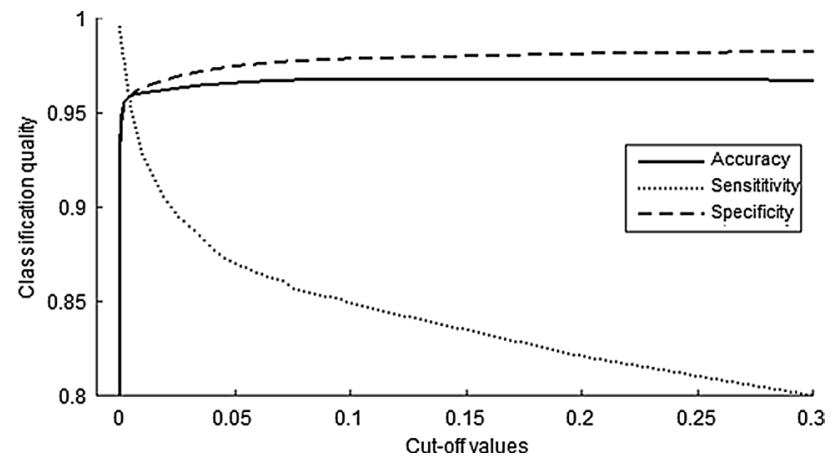

Fig. 5 The accuracy (solid line), sensitivity (dotted line), and specificity (dashed line) of classification plotted against cut-off values of the parameter $\mathrm{C}$, above which the algorithm would classify the patient as conscious. The plot is focused on the ranges of thresholds around the optimal threshold value $(\mathrm{C}=0.001)$ and the classification quality values relevant for that range of threshold cut-off values

just specific features of general anesthesia caused by sevoflurane (this study) or propofol [8].

In addition to the DTF, several other measures of connectivity have been suggested as a relevant markers for changes in the state or level of consciousness, including measures of transfer entropy [5, 35], directed coherence [36, 37], and Granger causality measures [38, 39]. Specifically, measures of the connectivity between frontal and parietal regions were found to differ between conscious and unconscious states, both for humans undergoing various forms of anesthesia [5, 35] or falling asleep [36], and for patients suffering from DOC [40]. For example, in a study using directed coherence-a measure closely related to DTFthe directionality of frontal-parietal functional connectivity covaried with NREM sleep stages and wakefulness [36]. However, approaches such as these (ours included), trying to quantify network properties based on passive observation of brain activity, are unlikely to justify strong conclusions about the underlying brain mechanisms [41]. Perturbational approaches, however, have indicated that a balanced interconnectivity between distant brain regions is likely to be crucial for maintaining a normal capacity for consciousness [4, 42-45]. Thus, it is plausible that reports from observational studies of altered connectivity related to loss of consciousness also often reflect relevant underlying changes.

The fact that filtering, rejection of artefacts, and other data cleaning techniques were deliberately avoided in this study (in order to simulate a setting relevant for real-time

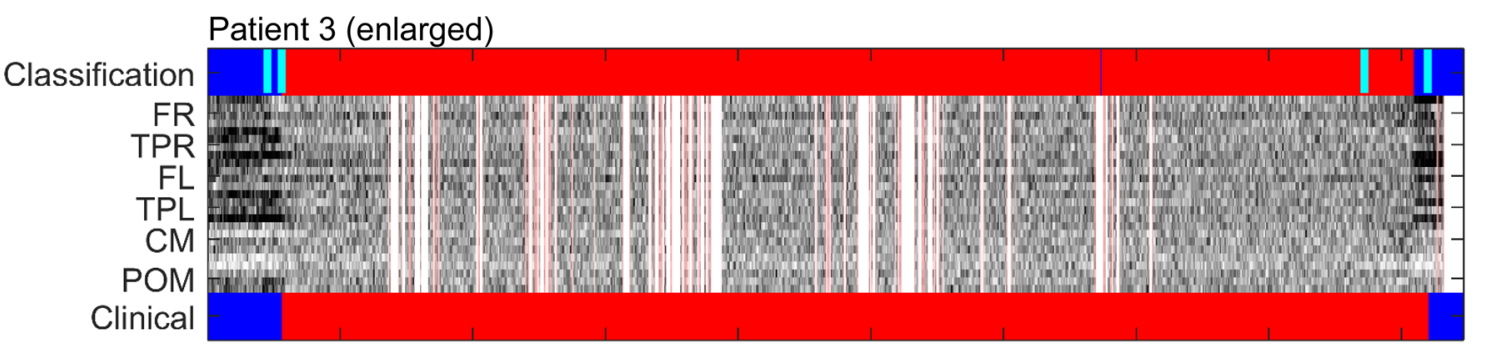

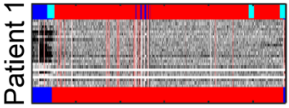

Total Time: $191 \mathrm{~min}$

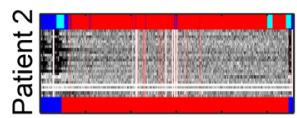

Total Time: $187 \mathrm{~min}$

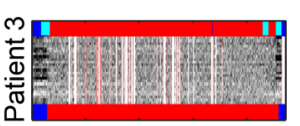

Total Time: $158 \mathrm{~min}$

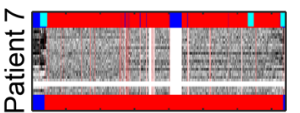

Total Time: $247 \mathrm{~min}$

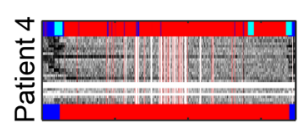

Total Time: 116 min

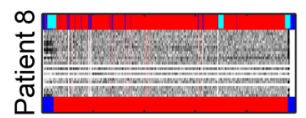

Total Time: 165 min

Total Time: $147 \mathrm{~min}$

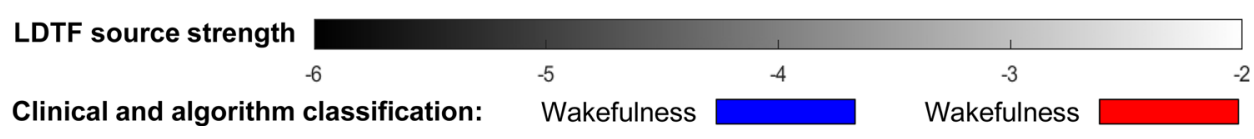

Fig. 6 Visualization of classification results for each patient. The time courses of DTF information source strengths for all patients are shown, together with their corresponding clinical judgement, and algorithmic classification, of their conscious state. The middle region of each panel, containing the information source values (LDTF) for every channel, follows the color scheme indicated in the color bar. In addition, epochs and channels marked as artefactual by the automatic data cleaning algorithm are marked with white columns and rows respectively. The bottom bar indicates the states of the patient reported by the clinical staff (blue: awake, red: anesthetized). The top bar represents the corresponding conscious state of the patient as classified by the algorithm (using the optimal threshold for classifying as awake, $\mathrm{C}>0.001$ ). In each panel, turquoise lines indicate the four main events in the anesthetic management: start of anesthesia administration, loss of verbal communication, stopping the anesthesia administration, and return of verbal communication. The top panel is just an enlarged version of patient 3's time course, included to give a better impression of the dynamics, as well as details in the classification 
monitoring of brain states in the clinic) may have further distorted the resulting connectivity matrices and yield faulty estimations of brain connectivity patterns. However, since the DTF is known to be robust to noise [46], and the median was used as a descriptive statistic for the mode of the distributions [47], this type of problems should be minimized [48]. Nevertheless, we remain agnostic regarding how the results reported here (regarding scalp level inferred connectivity) are related to the underlying changes in neural, effective connectivity within the brain. In fact, even with properly cleaned EEG data, the degree to which of sensor space estimates of connectivity are relevant for characterizing the underlying neural connectivity is disputed [49-51].

Furthermore, it is possible that our DTF measure may be influenced by changes in muscle activity related to anesthesia, as has previously been shown for the bispectral (BIS) index which is one of several methods used for monitoring depth of anesthesia [52]. Propofol is known to decrease muscle tone in the absence of neuromuscular blocking drugs [53], and it is uncertain to what extent such effects may contribute to the observed changes in DTF reported here and in our previous paper [8] as it may not be not possible with our methods to tease apart muscle relaxing effects from other effects anesthetics have on the brain and body. Similarly, sevoflurane is known to cause immobility [54], depress the excitability of motor neurons [55], and enhance the effect of neuromuscular blockers [56]. However, the degree to which sevoflurane affects muscle tonus and EMG contamination of the EEG signal under the conditions used in our study remains to be determined. Thus, we cannot exclude the possibility that the apparent changes in brain connectivity observed here, may at least partly result from changes in EMG contamination in the two states, rather than reflecting consciousness related changes within the brain. This is an issue that is hard to control for without additional pharmacological interventions that were not feasible in the clinical setting our data were obtained from. We did, however, avoid neuromuscular blockers when possible to minimize anesthesia related changes in EMG (only one patient (\#7) received an additional neuromuscular blocker (cisatrakurium, $14 \mathrm{mg}$ i.v.) due to problems related to intubation). However, to investigate this issue more directly, we are now initiating a follow-up study in which we will test the effect of pure neuromuscular block in the absence of anesthetics on measures assumed to track the depth of anesthesia.

Another possible limitation is that our choice of DTF as a measure was made for reasons not entirely grounded in theories of consciousness. This makes it more likely that the observed changes in connectivity are related to changes in state more generally (e.g. going from normal wakefulness to general anesthesia) rather than reflecting changes in the brain that are specifically and causally important for consciousness. However, taken together, our findings that similar patterns of DTF changes occur for at least two different types of anesthesia, combined with the previous findings of changes in DTF related to distinct states of consciousness, provide convergent evidence in support of a strong relation between consciousness and measures of brain connectivity [9-12], although further studies are needed to clarify whether and how the observed changes in our DTF-based measure are related to changes in consciousness as such.

Finally, other efforts are ongoing to test if our previous findings [8] can be reproduced in different experimental settings and under different conditions in order to assess the generalizability of the DTF-based measure as a marker of consciousness. However, these efforts have shown that reproduction is not always straight-forward, and that the particular topography of apparent information outflow may be sensitive to parameter choice in the analysis (e.g. filter types, sampling frequency, reference position, spatial filter functions) as well as the state of the person having their EEG measured. Furthermore, before measures such as the one discussed here can be used as clinical monitors of consciousness or anesthesia, studies aimed at understanding how they behave in transitions between coarse 'conscious' and 'unconscious' states must be carried out. Although we have shown that the DTF parameters abruptly change near transitions between 'awake' and 'anesthetized' states, there is significant variance in the exact time course of the classification confidence. The transitions should be studied experimentally outside of the clinic to allow even more precise quantification of the time of LOC and ROC, as well as allowing for intermittent awakenings with immediate reports about conscious experience (i.e. waking up the sleeping or sedated person with sound and/or touch stimuli in order to ask if they had any experience immediately before waking up; see for example work by Noreika et al. [20]), to understand whether the measures do in fact correctly identify changes in state of consciousness. Additionally, the clinical applicability of the methods applied in this paper is still questionable - not only because of uncertainties related to its classification accuracy, but also regarding the feasibility of routinely applying 25 EEG channel recordings to patients undergoing anesthesia and the computational costs associated with online application of the method. It would also be advisable to rigorously investigate the effects of precise pre-processing choices (e.g. reference position, sampling rate, presence and type of filters, artefact rejection) on DTF-parameters. This is because different practitioners may apply the measures in slightly different ways, with different equipment, and making different choices for preprocessing. It is not unlikely that such differences would change the resulting DTFparameters, which might affect the interpretation of the results. Thus, before drawing strong conclusions about the 
capacity of the method presented here and in our earlier publication to objectively track states of consciousness in humans, further studies are needed. In particular, studies with much larger sample sizes are required and investigations of the method's sensitivity to changing parameters (e.g. number of channels, sampling rate, and model order) as well as its response to other anesthetics (e.g. ketamine, xenon, and nitrous oxide).

\section{Conclusions}

Following our previous study where a DTF-based approach was used to detect changes in apparent brain connectivity during general anesthesia with propofol, we performed a follow-up study to validate our method by testing whether similar changes occur during loss of consciousness caused by another anesthetic, sevoflurane, which has partly different mechanisms of action. We found that our DTF-derived connectivity parameters showed similar changes in patients undergoing sevoflurane anesthesia as were previously observed with propofol anesthesia. These changes could once again be used to successfully classify the patients' state of anesthesia vs. wakefulness in accordance with the clinician's judgement, with accuracies and sensitivities exceeding $96 \%$, depending on the choice of cut-off value for our algorithm's confidence in classification. These results indicate that certain changes in DTF caused by general anesthesia generalize across at least two different anesthetics with partially distinct mechanisms of action. This can be regarded as further evidence in favor of brain connectivity being related to the level of consciousness in humans, although our study does not yet exclude the possibility that effect on muscle activity (EMG) may contaminate our EEG results. Thus, further studies are required for better understanding how the observed alterations in DTF are related to changes in brain state and consciousness.

Acknowledgements We would like to thank Frode Kolstad for being central in setting up the study, selecting patients and performing all the surgeries. Furthermore, we would like to thank Sally Rose and Marianne Nævra for their contributions to the study in mounting and recording the EEG, and handling of the data. This study was supported in part by the European Union's Horizon 2020 research and innovation programme under Grant Agreement 7202070 (Human Brain Project (HBP)) and the Norwegian Research Council (NRC: 262950/F20 and 214079/F20).

Funding Open Access funding provided by University of Oslo (incl Oslo University Hospital). This study was supported in part by the European Union's Horizon 2020 research and innovation programme under Grant Agreement 7202070 (Human Brain Project (HBP) and from the Norwegian Research Council (NRC: 262950/F20 and 214079/ F20).
Data availability Due to the sensitive nature of clinical data, the data are not shared publicly, but may be shared upon reasonable request from individuals.

Code availability The code used for running the analysis is available upon request.

\section{Compliance with ethical standards}

Conflict of interest The authors have no conflicts of interest to disclose.

Ethical approval The study was approved by the Regional Committee for Research Ethics (case number 2012/2015), and all patients included in the study signed a written consent form after oral and written information.

Informed consent Informed consent was obtained from all individual participants included in the study. The consent form signed by participants included information that results from the study would be published in a peer reviewed journal.

Open Access This article is licensed under a Creative Commons Attribution 4.0 International License, which permits use, sharing, adaptation, distribution and reproduction in any medium or format, as long as you give appropriate credit to the original author(s) and the source, provide a link to the Creative Commons licence, and indicate if changes were made. The images or other third party material in this article are included in the article's Creative Commons licence, unless indicated otherwise in a credit line to the material. If material is not included in the article's Creative Commons licence and your intended use is not permitted by statutory regulation or exceeds the permitted use, you will need to obtain permission directly from the copyright holder. To view a copy of this licence, visit http://creativecommons.org/licenses/by/4.0/.

\section{References}

1. Loomis AL, Harvey EN, Hobart GA. Cerebral states during sleep, as studied by human brain potentials. J Exp. 1937;21:127.

2. Purdon PL, Pierce ET, Mukamel EA, Prerau MJ, Walsh JL, Wong KFK, et al. Electroencephalogram signatures of loss and recovery of consciousness from propofol. Proc Natl Acad Sci USA. 2013;110:E1142-51.

3. Brazier MAB, Finesinger JE. Action of barbiturates on the cerebral cortex: electroencephalographic studies. Arch Neurol Psychiatry. 1945;53:51-8.

4. Casali AG, Gosseries O, Rosanova M, Boly M, Sarasso S, Casali $\mathrm{KR}$, et al. A theoretically based index of consciousness independent of sensory processing and behavior. Sci Transl Med. 2013;5:198ra105.

5. Lee U, Ku S, Noh G, Baek S, Choi B, Mashour GA. Disruption of frontal-parietal communication by ketamine, propofol, and sevoflurane. Anesthesiology. 2013;118:1264-75.

6. Schartner M, Seth A, Noirhomme Q, Boly M, Bruno M-A, Laureys $\mathrm{S}$, et al. Complexity of multi-dimensional spontaneous EEG decreases during propofol induced general anaesthesia. PLoS One. 2015; 10:e 0133532.

7. Mashour GA, Hudetz AG. Neural correlates of unconsciousness in large-scale brain networks. Trends Neurosci. 2018;41:150-60.

8. Juel BE, Romundstad L, Kolstad F, Storm JF, Larsson PG. Distinguishing anesthetized from awake state in patients: a new 
approach using one second segments of raw EEG. Front Hum Neurosci. 2018;12:40.

9. Höller Y, Thomschewski A, Bergmann J, Kronbichler M, Crone JS, Schmid EV, et al. Connectivity biomarkers can differentiate patients with different levels of consciousness. Clin Neurophysiol. 2014;125:1545-55.

10. Kamiński M, Blinowska K, Szclenberger W. Topographic analysis of coherence and propagation of EEG activity during sleep and wakefulness. Electroencephalogr Clin Neurophysiol. 1997;102:216-27.

11. De Gennaro L, Vecchio F, Ferrara M, Curcio G, Rossini PM, Babiloni C. Changes in fronto-posterior functional coupling at sleep onset in humans. J Sleep Res. 2004;13:209-17.

12. Bertini M, Ferrara M, De Gennaro L, Curcio G, Moroni F, Babiloni $\mathrm{C}$, et al. Directional information flows between brain hemispheres across waking, non-REM and REM sleep states: an EEG study. Brain Res Bull. 2009;78:270-5.

13. Chernik DA, Gillings D, Laine H, Hendler J, Silver JM, Davidson $\mathrm{AB}$, et al. Validity and reliability of the Observer's Assessment of Alertness/Sedation Scale: study with intravenous midazolam. J Clin Psychopharmacol. 1990;10:244-51.

14. Delorme A, Makeig S. EEGLAB: an open source toolbox for analysis of single-trial EEG dynamics including independent component analysis. J Neurosci Methods. 2004;134:9-21.

15. Schumacher EM, Stiris TA, Larsson PG. Effective connectivity in long-term EEG monitoring in preterm infants. Clin Neurophysiol. 2015;126:2261-8.

16. Eichler M. On the evaluation of information flow in multivariate systems by the directed transfer function. Biol Cybern. 2006;94:469-82.

17. Kamiński MJ, Blinowska KJ. A new method of the description of the information flow in the brain structures. Biol Cybern. 1991;65:203-10.

18. He B, Dai Y, Astolfi L, Babiloni F, Yuan H, Yang L. eConnectome: a MATLAB toolbox for mapping and imaging of brain functional connectivity. J Neurosci Methods. 2011;195:261-9.

19. Karniski W, Blair RC, Snider AD. An exact statistical method for comparing topographic maps, with any number of subjects and electrodes. Brain Topogr. 1994;6:203-10.

20. Noreika V, Jylhänkangas L, Móró L, Valli K, Kaskinoro K, Aantaa $\mathrm{R}$, et al. Consciousness lost and found: subjective experiences in an unresponsive state. Brain Cogn. 2011;77:327-34.

21. Leslie K. Awareness and dreaming during TIVA. In: Absalom AR, Mason KP, editors. Total intravenous anesthesia and target controlled infusions: a comprehensive global anthology. Cham: Springer International Publishing; 2017. p. 783-796.

22. Sarasso S, Boly M, Napolitani M, Gosseries O, Charland-Verville $\mathrm{V}$, Casarotto S, et al. Consciousness and complexity during unresponsiveness induced by propofol, xenon, and ketamine. Curr Biol. 2015;25:3099-105

23. Storm JF, Boly M, Casali AG, Massimini M, Olcese U, Pennartz CMA, et al. Consciousness regained: disentangling mechanisms, brain systems, and behavioral responses. J Neurosci. 2017;37:10882-93.

24. Alkire MT, Hudetz AG, Tononi G. Consciousness and anesthesia. Science. 2008;322:876-80.

25. Rudolph U, Antkowiak B. Molecular and neuronal substrates for general anaesthetics. Nat Rev Neurosci. 2004;5:709-20.

26. Hudetz AG, Mashour GA. Disconnecting consciousness: is there a common anesthetic end point? Anesth Analg. 2016;123:1228.

27. Antkowiak B. Different actions of general anesthetics on the firing patterns of neocortical neurons mediated by the GABAAReceptor. Anesthesiology. 1999;91:500-11.

28. Gugino LD, Chabot RJ, Prichep LS, John ER, Formanek V, Aglio LS. Quantitative EEG changes associated with loss and return of consciousness in healthy adult volunteers anaesthetized with propofol or sevoflurane. Br J Anaesth. 2001;87:421-8.

29. Akeju O, Westover MB, Pavone KJ, Sampson AL, Hartnack KE, Brown EN, et al. Effects of sevoflurane and propofol on frontal electroencephalogram power and coherence. Anesthesiology. 2014;121:990-8.

30. Blain-Moraes S, Tarnal V, Vanini G, Alexander A, Rosen D, Shortal B, et al. Neurophysiological correlates of sevofluraneinduced unconsciousness. Anesthesiology. 2015;122:307-16.

31. Jeong YB, Kim JS, Jeong SM, Park JW, Choi IC. Comparison of the effects of sevoflurane and propofol anaesthesia on regional cerebral glucose metabolism in humans using positron emission tomography. J Int Med Res. 2006;34:374-84.

32. Banoub M, Tetzlaff JE, Schubert A. Pharmacologic and physiologic influences affecting sensory evoked potentials: implications for perioperative monitoring. Anesthesiology. 2003;99:716-37.

33. Boisseau N, Madany M, Staccini P, Armando G, Martin F, Grimaud $\mathrm{D}$, et al. Comparison of the effects of sevoflurane and propofol on cortical somatosensory evoked potentials. Br J Anaesth. 2002;88:785-9.

34. Blinowska KJ. Review of the methods of determination of directed connectivity from multichannel data. Med Biol Eng Comput. 2011;49:521-9.

35. Ku S-W, Lee U, Noh G-J, Jun I-G, Mashour GA. Preferential inhibition of frontal-to-parietal feedback connectivity is a neurophysiologic correlate of general anesthesia in surgical patients. PLoS One. 2011;6:e25155.

36. Lioi G, Bell SL, Smith DC, Simpson DM. Directional connectivity in the EEG is able to discriminate wakefulness from NREM sleep. Physiol Meas. 2017;38:1802-20.

37. Maksimow A, Silfverhuth M, Långsjö J, Kaskinoro K, Georgiadis S, Jääskeläinen S, et al. Directional connectivity between frontal and posterior brain regions is altered with increasing concentrations of propofol. PLoS One. 2014;9:e113616.

38. Barrett AB, Murphy M, Bruno M-A, Noirhomme Q, Boly M, Laureys $\mathrm{S}$, et al. Granger causality analysis of steady-state electroencephalographic signals during propofol-induced anaesthesia. PLoS One. 2012;7:e29072.

39. Seth AK, Barrett AB, Barnett L. Causal density and integrated information as measures of conscious level. Philos Trans A Math Phys Eng Sci. 2011;369:3748-67.

40. Boly M, Massimini M, Garrido MI, Gosseries O, Noirhomme Q, Laureys $\mathrm{S}$, et al. Brain connectivity in disorders of consciousness. Brain Connect. 2012;2:1-10.

41. Massimini M, Boly M, Casali A, Rosanova M, Tononi G. A perturbational approach for evaluating the brain's capacity for consciousness. Prog Brain Res. 2009;177:201-14.

42. Massimini M, Ferrarelli F, Huber R, Esser SK, Singh H, Tononi G. Breakdown of cortical effective connectivity during sleep. Science. 2005;309:2228-32.

43. Ferrarelli F, Massimini M, Sarasso S, Casali A, Riedner BA, Angelini G, et al. Breakdown in cortical effective connectivity during midazolam-induced loss of consciousness. Proc Natl Acad Sci USA. 2010;107:2681-6.

44. Rosanova M, Gosseries O, Casarotto S, Boly M, Casali AG, Bruno M-A, et al. Recovery of cortical effective connectivity and recovery of consciousness in vegetative patients. Brain. 2012;135:1308-20.

45. Casarotto S, Comanducci A, Rosanova M, Sarasso S, Fecchio M, Napolitani M, et al. Stratification of unresponsive patients by an independently validated index of brain complexity. Ann Neurol. 2016;80:718-29.

46. Astolfi L, Cincotti F, Mattia D, Marciani MG, Baccala LA, de Vico FF, et al. Comparison of different cortical connectivity estimators for high-resolution EEG recordings. Hum Brain Mapp. 2007;28:143-57. 
47. Dukic S, Iyer PM, Mohr K, Hardiman O, Lalor EC, Nasseroleslami B. Estimation of coherence using the median is robust against EEG artefacts. In: Annual International Conference of the IEEE Engineering in Medicine and Biology Society. 2017. p. 3949-3952.

48. Schumacher EM, Westvik AS, Larsson PG, Lindemann R, Westvik J, Stiris TA. Feasibility of long-term continuous EEG monitoring during the first days of life in preterm infants: an automated quantification of the EEG activity. Pediatr Res. 2011;69:413-7.

49. Papadopoulou M, Friston K, Marinazzo D. Estimating directed connectivity from cortical recordings and reconstructed sources. Brain Topogr. 2019;32(4):741-52.

50. Brunner C, Billinger M, Seeber M, Mullen TR, Makeig S. Volume conduction influences scalp-based connectivity estimates. Front Comput Neurosci. 2016;10:121.

51. Kaminski M, Blinowska KJ. The influence of volume conduction on DTF estimate and the problem of its mitigation. Front Comput Neurosci. 2017;11:36.

52. Schuller PJ, Newell S, Strickland PA, Barry JJ. Response of bispectral index to neuromuscular block in awake volunteers. $\mathrm{Br}$ J Anaesth. 2015;115(Suppl 1):i95-i103.
53. Haeseler G, Störmer M, Bufler J, Dengler R, Hecker H, Piepenbrock $\mathrm{S}$, et al. Propofol blocks human skeletal muscle sodium channels in a voltage-dependent manner. Anesth Analg. 2001;92:1192.

54. King BS, Rampil IJ. Anesthetic depression of spinal motor neurons may contribute to lack of movement in response to noxious stimuli. Anesthesiology. 1994;81:1484-92.

55. Zhou HH, Mehta M, Leis AA. Spinal cord motoneuron excitability during isoflurane and nitrous oxide anesthesia. Anesthesiology. 1997;86:302-7.

56. Wulf H, Kahl M, Ledowski T. Augmentation of the neuromuscular blocking effects of cisatracurium during desflurane, sevoflurane, isoflurane or total i.v. anaesthesia. Br J Anaesth. 1998;80:308-12.

Publisher's Note Springer Nature remains neutral with regard to jurisdictional claims in published maps and institutional affiliations. 